\title{
Insights into internal dynamics of 6-phosphogluconolactonase from Trypanosoma brucei studied by nuclear magnetic resonance and molecular dynamics
}

\author{
Paolo A. Calligari, ${ }^{1,2,3}$ Gilmar F. Salgado, ${ }^{1,2,3^{\star}}$ Philippe Pelupessy, ${ }^{1,2,3}$ Philippe Lopes, ${ }^{4}$ \\ Jamal Ouazzani, ${ }^{4}$ Geoffrey Bodenhausen, ${ }^{1,2,3,5^{\star}}$ and Daniel Abergel ${ }^{1,2,3}$ \\ ${ }^{1}$ Département de Chimie, Ecole Normale Supérieure, 24 rue Lhomond, 75231 Paris Cedex 05, France \\ ${ }^{2}$ Université Pierre-et-Marie Curie, Place Jussieu, 75005 Paris, France \\ ${ }^{3}$ UMR 7203 CNRS, 24 rue Lhomond, 75231 Paris Cedex 05, France \\ ${ }^{4}$ Institut de Chimie des Substances Naturelles, UPR 2301 CNRS, avenue de la Terrasse, 91198 Gif sur Yvette Cedex, France \\ ${ }^{5}$ Institut de Sciences et Ingénierie Chimiques, Ecole Polytechnique Fédérale de Lausanne, Batochime, 1015 Lausanne, \\ Switzerland
}

\begin{abstract}
Nuclear magnetic resonance is used to investigate the backbone dynamics in 6-phosphogluconolactonase from Trypanosoma brucei (Tb6PGL) with (holo-) and without (apo-) 6phosphogluconic acid as ligand. Relaxation data were analyzed using the model-free approach and reduced spectral density mapping. Comparison of predictions, based on $77 \mathrm{~ns}$ molecular dynamics simulations, with the observed relaxation rates gives insight into dynamical properties of the protein and their alteration on ligand binding. Data indicate dynamics changes in the vicinity of the binding site. More interesting is the presence of perturbations located in remote regions of this well-structured globular protein in which no large-amplitude motions are involved. This suggests that delocalized changes in dynamics that occur upon binding could be a general feature of protein-target interactions.
\end{abstract}

Proteins 2012; 80:1196-1210. (C) 2011 Wiley Periodicals, Inc.

Key words: NMR; relaxation; protein dynamics; 6-phospho-gluconolactonase (6PGL); molecular dynamics simulations (MD).

\section{INTRODUCTION}

Internal mobility is recognized as a crucial factor of protein function. Relevant motions occur over a wide range of time scales from $10^{-12} \mathrm{~s}$ to a few seconds 1,2 and can be of various kinds, such as local fluctuations (e.g., ring flips, cis/trans isomerization, etc.), or large-amplitude motions, which may allow for fine tuning of the three-dimensional (3D) structure during interactions and catalytic processes. Nuclear magnetic resonance (NMR) spectroscopy has contributed to shed light on molecular aspects of enzymatic activity (for recent reviews, see Refs. 3 and 4).

In this work, we have used NMR spectroscopy combined with molecular dynamics (MD) to investigate the effects of ligand binding on the internal dynamics of the enzyme 6-phosphogluconolactonase (Tb6PGL) of the parasite Trypanosoma brucei, responsible for the African sleeping sickness. The enzyme 6PGL is one of the six enzymes of the pentose phosphate pathway (PPP) of T. brucei, and hydrolyzes $\delta$-6-phosphogluconolactone to 6-phosphogluconic acid (6PGA). Tb6PGL plays a key role during infection, by providing the important reductive agent NADPH, and forms ribose 5-phosphate, a major nucleic acid precursor, as well as several metabolic intermediates, such as fructose 6-phosphate and glyceraldehyde 3-phosphate. ${ }^{5,6}$ Enzymes that play a role in glycolysis or in the PPP are potential drug targets against T. brucei, because several of these enzymes are found in the glycosome, an organelle which, in contrast to the situation in most

Additional Supporting Information may be found in the online version of this article.

Abbreviations: $M D$, molecular dynamics; NMR, nuclear magnetic resonance; NOE, nuclear Overhauser enhancement; $R_{\mathrm{ex}}$, exchange contribution to transverse relaxation rate $R_{2} ; S^{2}$, generalized order parameter (reflects motions on the ps-ns time scale); $T_{1}$, longitudinal ("spin-lattice") relaxation time constant $\left(T_{1}=1 / R_{1}\right) ; T_{2}$, transverse relaxation time constant $\left(T_{2}=1 / R_{2}\right) ; \tau_{\mathrm{i}}$, correlation time for internal motions on the ps-ns time scale; 6PGA, 6-phosphogluconic acid (=ligand); 6PGL, 6-phosphogluconolactonase (=enzyme); Tb6PGL, 6PGL from T. brucei; apo-Tb6PGL, Tb6PGA without ligand; holo-Tb6PGL, complex of Tb6PGL with 6PGA as ligand.

Grant sponsor: CNRS (postdoctoral fellowship).

Paolo A. Calligari and Gilmar F. Salgado contributed equally to this work

${ }^{\star}$ Correspondence to: Gilmar F. Salgado, Laboratoire ARNA (INSERM U869 - Université Bordeaux Segalen), Institut Européen de Chimie et Biologie, 2 rue Robert Escarpit, 33076 Pessac Cedex, France. E-mail: g.salgado@iecb.ubordeaux.fr or to: Geoffrey Bodenhausen, Département de Chimie, Ecole Normale Supérieure, 24 rue Lhomond, 75231 Paris Cedex 05, France. E-mail: Geoffrey.Bodenhausen@ens.fr.

Received 5 July 2011; Revised 23 November 2011; Accepted 4 December 2011

Published online 22 December 2011 in Wiley Online Library (wileyonlinelibrary.com). DOI: 10.1002/prot.24019 
eukaryotic cells, is separated from the cytosol. This may entail significant differences with respect to the mammalian host, thus enabling the design of specific drugs, ${ }^{7,8}$ as successfully demonstrated in the case of NADH-fumarate reductase and other systems. ${ }^{9,10}$ For these reasons, any progress toward a deeper understanding of the catalytic mechanism and of the dynamic changes in the protein that occur upon substrate binding and catalysis is clearly desirable.

In this article, we investigate dynamic changes occurring in the protein on ligand binding using ${ }^{15} \mathrm{~N}$ NMR relaxation measurements of the free protein and its complex with the reaction product 6PGA. We show that ligand binding leads to changes of internal dynamics of the protein in various regions. Interestingly, many of the affected residues are remote from the active site, and although they are not directly involved in catalysis, they are clearly affected by a redistribution of the dynamics across the protein, which occurs on ligand binding. Our results are analyzed in the light of previous X-ray and docking studies. ${ }^{11}$

A number of observations ${ }^{12-15}$ support the idea that proteins comprise dynamical networks that can regulate thermodynamics of protein-ligand interactions. In this respect, this work provides additional evidence for the role of dynamics. Moreover, the relationships between local dynamics and catalytic mechanisms remain in general largely unknown. These aspects, despite an increasing number of studies, constitute a stimulating and open question in this field. ${ }^{16}$

\section{MATERIALS AND METHODS}

\section{Sample preparation}

Expression and purification of uniformly ${ }^{2} \mathrm{H},{ }^{13} \mathrm{C}$, and ${ }^{15} \mathrm{~N}$-labeled recombinant Tb6PGA was performed as described elsewhere. ${ }^{6}$ The sample was dialyzed for $48 \mathrm{~h}$ using $1 \mathrm{~mL}$ Float-A-Lyzer dialysis membranes (SpectrumLabs) with a MWCO of $10 \mathrm{kDa}$, against $20 \mathrm{mM}$ phosphate buffer at pH 6.1 containing $120 \mathrm{mM} \mathrm{NaCl}$, with a final concentration of about $0.55 \mathrm{mM}$ determined by $\mathrm{UV} / \mathrm{Vis}$ spectroscopy at $280 \mathrm{~nm}$ with an extinction coefficient $\varepsilon=21,095 \mathrm{M}^{-1} \mathrm{~cm}^{-1}$. The $3 \mathrm{~mm}$ NMR sample tube was filled to about $200 \mu \mathrm{L}$ by adding $\mathrm{D}_{2} \mathrm{O} / \mathrm{H}_{2} \mathrm{O}$ $(10 \% / 90 \%)$. The concentrations of the protein $6 \mathrm{PGL}$ and of the ligand 6PGA were approximately 0.55 and $0.66 \mathrm{M}$.

\section{Relaxation measurements of backbone ${ }^{15} \mathrm{~N}$ nuclei and analysis}

The longitudinal and transverse relaxation rates $R_{1}$ and $R_{2}$ and the steady-state ${ }^{15} \mathrm{~N}\left\{{ }^{1} \mathrm{H}\right\}$ nuclear Overhauser enhancement (NOE) $\eta$ were measured at $290 \mathrm{~K}$ using $2 \mathrm{D}$ experiments employing the principles of transverse relaxation optimized spectroscopy (TROSY). ${ }^{17}$ The experiments were performed on a Bruker Avance $900 \mathrm{MHz}$ spectrometer $\left(B_{0}=21.1 \mathrm{~T}\right)$ equipped with a cryogenically cooled probe. The spectral widths were $14.36 \mathrm{kHz}$ for ${ }^{1} \mathrm{H}$ and $3.10 \mathrm{kHz}$ for ${ }^{15} \mathrm{~N}$. The ${ }^{15} \mathrm{~N} R_{1}$ rates were obtained using recovery delays of 50,150, 250, 500, 750, 1000, 1500, 2000, and 2500 ms. The Carr-Purcell Meiboom-Gill sequences ${ }^{18,19}$ performed to determine the ${ }^{15} \mathrm{~N} R_{2}$ rates used, on the nitrogen channel, $180^{\circ}$ pulses spaced by $2 \tau=1 \mathrm{~ms}$, and overall durations $8.16,16.32$ (twice), 24.48, 32.64, 48.96, $65.28,81.6,97.92$, and $114.24 \mathrm{~ms}$. Usually 16 scans were used per increment, and $256\left({ }^{15} \mathrm{~N}\right) \times 2048\left({ }^{1} \mathrm{H}\right)$ complex points were collected for each experiment in a interleaved manner. Recycle delays of $2.5 \mathrm{~s}$ were used for both $R_{1}$ and $R_{2}$ experiments. The ${ }^{15} \mathrm{~N}\left\{{ }^{1} \mathrm{H}\right\}$ NOE experiments were performed with and without saturation of the protons during a recycle delay of 12 s. $^{20}$ Resonance assignments at field strengths $B_{0}=21.1$ and 21.1 Twere obtained using modified TROSY-based HNCA, HN(CO)CA, HN(CA)CB, HNCO, 21 and HNCANH experiments. 22,23

All 3D spectra were processed with the NMRPipe/ NMRDraw package. ${ }^{24}$ The signals were apodized by cosine-bell and squared cosine-bell functions in the indirect and direct dimensions. Digital resolution in the indirect dimension was doubled through linear prediction. All 2D spectra were processed with Topspin (Bruker). The 2D peak heights and volumes were determined using Sparky. 25 The $R_{1}$ and $R_{2}$ rates were determined by fitting the peak heights to exponential functions $I(t)=I_{0} \exp \left(-R_{1,2} t\right)$, using a nonlinear least squares analysis where Gaussian random errors were added to the experimental signal amplitudes and averaged over 100 fits. Steady-state NOE values were determined from the ratios of the peak intensities with and without saturation of the protons. Errors in NOE values were determined by error propagation based on the root-mean-square of the noise in the spectra.

Overall rotational correlation times were estimated using HYDRONMR ${ }^{26}$ and TENSOR2, ${ }^{27}$ based on the ratios $R_{1} / R_{2}$, using only the most rigid residues. Modelfree (MF) analysis was performed with the TENSOR2 software. ${ }^{27}$ Five models of increasing complexity were used to determine the generalized order parameter $S^{2}$, the effective correlation time $\tau_{\mathrm{i}}$ for motions of the backbone $\mathrm{N}-\mathrm{H}$ vectors, the chemical exchange contribution $R_{\mathrm{ex}}$, and an additional order parameter $S_{\mathrm{f}}^{2}$ that reflects contributions to the spectral density due to fast motions with $\tau_{\mathrm{i}}<20$ ps. ${ }^{3,18}$ Model 1 considers only $S^{2}$; Model 2 has two parameters $S^{2}$ and $\tau_{\mathrm{i}}$; Model 3 gives $S^{2}$ and $R_{\mathrm{ex}}$; Model 4 has three parameters $S^{2}, \tau_{\mathrm{i}}$, and $R_{\mathrm{ex}}$; and finally Model $5^{28}$ provides $S_{\mathrm{s}}^{2}, S_{\mathrm{f}}^{2}$, and $\tau_{\mathrm{i}}$, with $S^{2}=S_{\mathrm{s}}^{2} S_{\mathrm{f}}^{2}$. Typically, 200-500 Monte Carlo steps were used to minimize the target function:

$\chi^{2}=\sum_{i} \frac{\left(R_{1 i}^{\text {exp }}-R_{1 i}^{\text {calc }}\right)^{2}}{\sigma_{R_{1 i}^{2}}}+\frac{\left(R_{2 i}^{\text {exp }}-R_{2 i}^{\text {calc }}\right)^{2}}{\sigma_{R_{2 i}^{2}}}+\frac{\left(\eta_{i}^{\exp }-\eta_{i}^{\text {calc }}\right)^{2}}{\sigma_{\eta_{i}^{2}}}$

The following residues could not be fitted in a satisfying manner: N89, D98, F105, T219, G229, L255, in the apo- 
protein, and E15, I40, Y52, A53, N61, L62, G74, D75, V79, R125, V126, A129, K132, Q133, V152, G164, V184, R200, S204, A206, A211, I215, W225, G229, S252, and L255, A260 in the holo-complex.

The relations between the ${ }^{15} \mathrm{~N}$ relaxation rates and the spectral densities $J(\omega)$ are given by the following equations: 29

$$
R_{1}=\frac{d^{2}}{4}\left[J\left(\omega_{H}-\omega_{N}\right)+3 J\left(\omega_{N}\right)+6 J\left(\omega_{H}+\omega_{N}\right)\right]+c^{2} J\left(\omega_{N}\right)
$$

$$
\begin{aligned}
R_{2}= & \frac{d^{2}}{8}\left[4 J(0)+J\left(\omega_{H}-\omega_{N}\right)+3 J\left(\omega_{N}\right)+6 J\left(\omega_{H}\right)\right. \\
& \left.+6 J\left(\omega_{H}+\omega_{N}\right)\right]+\frac{c^{2}}{6}\left[3 J\left(\omega_{N}\right)+4 J(0)\right]+R_{\mathrm{ex}} \\
\eta= & 1+\frac{d^{2}}{4} \frac{\gamma_{H}}{\gamma_{N}}\left[6 J\left(\omega_{H}+\omega_{N}\right)-J\left(\omega_{H}-\omega_{N}\right)\right] / R_{1}
\end{aligned}
$$

where $d=\left[\mu_{\mathrm{o}} h \gamma_{\mathrm{N}} \gamma_{\mathrm{H}} /\left(8 \pi^{2}\right)\right]\left(1 /<r_{\mathrm{NH}}^{3}>\right), c=\omega_{\mathrm{N}} \Delta \tilde{\sigma} \sqrt{3}$, $\mu_{\mathrm{o}}=4 \pi \times 10^{-7} \mathrm{~N} / \mathrm{A}^{2}$ is the permeability in vacuum, $\gamma_{\mathrm{H}}$ $=2.6752 .10^{8} \mathrm{rad} \mathrm{s}^{-1} \mathrm{~T}^{-1}$ and $\gamma_{\mathrm{N}}=-2.713 .10^{7} \mathrm{rad} \mathrm{s}^{-1}$ $\mathrm{T}^{-1}$ are the gyromagnetic ratios of ${ }^{1} \mathrm{H}$ and ${ }^{15} \mathrm{~N}, \omega_{\mathrm{H}}$ and $\omega_{\mathrm{N}}$ are the Larmor frequencies of these nuclei (in rad $\left.\mathrm{s}^{-1}\right), h=6.626 \times 10^{-34} \mathrm{Js}, r_{\mathrm{NH}}=1.02 \AA$ is the average amide bond length, and $\Delta \sigma=-160 \mathrm{ppm}$ is the average chemical shift anisotropy of amide ${ }^{15} \mathrm{~N}$ nuclei. In its simplest implementation, the reduced spectral density mapping $(\mathrm{SDM})^{30}$ approach assumes that high-frequency motions are characterized by a nearly "white" spectral density function with $J\left(\omega_{\mathrm{H}}-\omega_{\mathrm{N}}\right) \approx J\left(\omega_{\mathrm{H}}\right) \approx J\left(\omega_{\mathrm{H}}+\right.$ $\left.\omega_{\mathrm{N}}\right)$, and that one may consider a single value of the spectral density function at $\omega_{\mathrm{h}}=0.87 \omega_{\mathrm{H}}$, where $J(\omega)$ denotes the exact spectral density function. With this assumption, the number of unknown values of the $a p$ proximate spectral density function $J_{\text {red }}(\omega)$ in Eqs. (2)(4) is reduced to three. These can be inverted to yield $J_{\text {red }}\left(\omega_{\mathrm{h}}\right), J_{\text {red }}\left(\omega_{N}\right)$ and $J_{\text {red }}(0)$. Calculations were performed through routines written in Mathematica (Wolfram Research, Champaign, IL), using nonlinear global optimization algorithms.

\section{MD simulations}

MD simulations up to $t_{\mathrm{MD}}=77 \mathrm{~ns}$ were performed using the NAMD program ${ }^{31}$ with the all-atom force field AMBER99SB $^{32}$ and periodic boundary conditions for both apo-Tb6 PL and its holo-form in complex with 6PGA, taking as initial configurations their structures from the Protein Data Bank (PDB) (entries 2JOE and $3 E 7 F)$. For $a p o-T b 6 \mathrm{PGL}$, a total of $9090 \mathrm{SPC} / \mathrm{E}$ water molecules and seven $\mathrm{Na}^{+}$ions were included, resulting in an electrically neutral system of 31,264 atoms. In the case of holo-Tb6PGL, 10,217 SPC/E water molecules were included so that the system comprised 34,632 atoms. In the active site of holo-Tb6PGL (see Fig. S2 in Supporting Information), the side chains of residues R200, R77, and $\mathrm{H} 165$ were protonated. The $\mathrm{Zn}^{2+}$ ion was removed from the $3 \mathrm{E} 7 \mathrm{~F}$ structure because it has no effect on the active site dynamics and on the function of the protein. ${ }^{11}$ The force field parameters for the ligand 6PGA were those of the Generic Amber Force Field. ${ }^{33}$ Electrostatic interactions were computed by using the particle mesh Ewald method $^{34}$ with a cut-off of $12 \AA$. The lengths of all bonds involving hydrogen atoms in the protein were fixed and treated by the SHAKE algorithm, 35 thus permitting us to increase the integration time step to $2 \mathrm{fs}$. After preliminary minimization starting with the PDB structure, all simulations were first equilibrated at constant temperature $(290 \mathrm{~K})$ and constant pressure (1 bar) using a Langevin thermostat ${ }^{36}$ coupled with a NoséHoover barostat. ${ }^{37}$ The equilibrated complexes were used as starting points of 77 ns runs. The RMSD values with respect to the initial structure remained below $1.5 \AA$, as expected for a stable protein (Fig. S1 in Supporting Information).

The generalized order parameter $S^{2}$ was calculated from MD trajectories using the expression:

$$
S^{2}=\frac{4 \pi}{5} \sum_{|m|<2}\left|\left\langle Y_{2 m}(\theta, \phi)\right\rangle\right|^{2}
$$

where $Y_{2 \mathrm{~m}}$ are spherical harmonics, and where the ensemble averages can be replaced in practice by time averages over the total length of the simulations.

Assuming statistical decoupling of internal motions and isotropic overall tumbling, one can decompose $C(t)$ in terms of the overall $C_{\mathrm{O}}(t)=\exp \left(-t / \tau_{\mathrm{c}}\right)$ and internal $C_{\mathrm{I}}(t)$ correlation functions.

$$
\left.C(t)=C_{0}(t) \times C_{I}(t)=\exp \left(-t / \tau_{c}\right)\left\langle P_{2} \cos [\mathbf{u}(t) \cdot \mathbf{u}(0)]\right)\right\rangle
$$

where $P_{2}$ is the second-rank Legendre polynomial and the unit vector $\mathbf{u}$ describes the orientation of the $\mathrm{N}-\mathrm{H}$ bond vector in the molecular reference frame. In practice, because of the finite length of MD trajectories, the internal correlation function was partitioned as 32,38 .

$$
\begin{aligned}
J(\omega) \approx & \int_{0}^{t_{\max }} C_{I}(t) C_{0}(t) \cos \omega t d t \\
& +\int_{t_{\max }}^{\infty} C_{I \infty} C_{0}(t) \cos \omega t d t
\end{aligned}
$$

where $t_{\max }$ represents the maximum interval over which the correlation function can be calculated with sufficiently good statistics. It is customarily fixed to $10 \%$ of the overall length of the trajectory, ${ }^{39}$ so that $t_{\max } \approx 7.7$ ns in our simulations. Moreover, $C_{\mathrm{I} \infty} \approx S_{\mathrm{MD}}^{2}$ if the following condition is met: 


$$
\left|C_{I}\left(t_{\max }\right)-S_{M D}^{2}\right| \leq 0.01
$$

where $C_{\mathrm{I}}\left(t_{\max }\right)$ is the average value over the last 20 ps of the correlation function. If Eq. (8) is not verified, the value of $C_{\mathrm{I}}\left(t_{\max }\right)$ is used. However, in this case, internal motions with time scales longer than $t_{\max }$ may likely affect the value of the spectral density functions.

\section{RESULTS AND DISCUSSION}

\section{Relaxation data}

The protein Tb6PGL comprises a total of 266 residues. Longitudinal and transverse ${ }^{15} \mathrm{~N}$ backbone relaxation rates $\left(R_{1}\right.$ and $\left.R_{2}\right)$ and steady-state ${ }^{15} \mathrm{~N}-\left\{{ }^{1} \mathrm{H}\right\}$ NOE's of apo- and holo-Tb6PGL were measured at 21.1 T (Fig. 1). Due to the presence of 16 proline residues that lack $\mathrm{H}^{\mathrm{N}}$ protons, and a number of spectral overlaps, only 239 residues could be analyzed. For a few residues (L16, S46, T51, D87, and L157), data analysis was only tentative, due to a low signal/noise ratio $(\mathrm{S} / \mathrm{N}<10)$. Chemical shift assignments of backbone residues were in agreement with previous reports. 5,40

The $R_{2}$ rates lie in the intervals $7.8 \pm 0.31<R_{2}<$ $54.5 \pm 13.4 \mathrm{~s}^{-1}$ for apo-Tb6PGL and $7.8 \pm 0.06<R_{2}<$ $54.5 \pm 15.3 \mathrm{~s}^{-1}$ for holo-Tb6PGL. The $R_{1}$ rates are distributed within the ranges $0.275 \pm 0.017<R_{1}<1.22 \pm$ $0.026 \mathrm{~s}^{-1}$ for apo-Tb6PGL and $0.25 \pm 0.026<R_{1}<1.25$ $\pm 0.067 \mathrm{~s}^{-1}$ for the holo-form. Finally, the average ${ }^{15} \mathrm{~N}$ $\left\{{ }^{1} \mathrm{H}\right\}$ NOE values are $0.79 \pm 0.14$ for apo-Tb6PGL and $0.79 \pm 0.13$ for the holo-form. The lowest NOE value of $\sim 0.18$ was observed for D192. Some NOE values exceed the expected maximum for a protein of this size. However, because $\sim 70 \%$ of the residues exceeded this threshold by less than one standard deviation $\sigma$ and $94 \%$ were within $2 \sigma$, these results were overall statistically satisfactory. The $R_{1}$ and $R_{2}$ relaxation rates as well as the NOE's show significant variations in flexible regions, including the C-terminus and loops. This can be seen in Figure 1, where significantly higher $R_{1}$, lower $R_{2}$, and lower NOE values in the $\mathrm{C}$-terminus and loops indicate the presence of increased mobility in these parts of the protein backbone.

\section{MF analysis}

The correlation times of overall tumbling were estimated to be $\tau_{\mathrm{c}}^{\text {apo }}=19.9 \pm 0.3 \mathrm{~ns}$ and $\tau_{\mathrm{c}}^{\text {holo }}=19.7 \pm$ 0.3 ns by using trimmed $R_{2} / R_{1}$ ratios of the most rigid residues $^{41}$ after discarding the $20 \%$ most extreme values. Estimates calculated using HYDRONMR ${ }^{26}$ gave $\tau_{\mathrm{c}}^{\text {apo }}=$ 19.4 and $\tau_{\mathrm{c}}^{\text {holo }}=19.5 \mathrm{~ns}$, in good agreement with the experimental values. The diffusion tensor estimated by HYDRONMR led to $D_{\mathrm{z}}^{\text {apo }}=9.2 \times 10^{6}, D_{\mathrm{y}}^{\text {apo }}=8.3 \times$ $10^{6}, D_{\mathrm{x}}^{\text {apo }}=8.7 \times 10^{6}, D_{\mathrm{z}}^{\text {holo }}=9.2 \times 10^{6}, D_{\mathrm{y}}^{\text {holo }}=8.1$ $\times 10^{6}, D_{\mathrm{x}}^{\text {holo }}=8.6 \times 10^{6} \mathrm{~s}^{-1}$. This allowed us to neglect the rhombicity, because $\left(D_{\mathrm{x}}-D_{\mathrm{y}}\right) / 2 D_{\mathrm{z}}<2 \%$, and the anisotropy, because $2 D_{z} /\left(D_{\mathrm{x}}+D_{\mathrm{y}}\right)<1.1$ for apo- and holo-forms of the protein. This was in agreement with the analysis of overall diffusion from relaxation data using TENSOR2 software, which did not provide any statistically significant diffusion anisotropy.

Internal mobility was analyzed using the MF approach, $, 3,18,28,42$ as summarized in Figures 2 and 3. Differences between order parameters on ligand binding were in general rather small, and were considered significant only if there is no overlap between error bars. Modifications of order parameters induced by ligand binding are scattered across the entire backbone of the protein, rather than localized near the active site. Indeed, increased mobility on binding $\left(\Delta S^{2}=S^{2 \text { holo }}-S^{\text {2apo }} \leq\right.$ -0.1) was observed for residues L16, A41, E76, R77, E145, V155, S168, L217, and A235, whereas the opposite trend $\left(\Delta S^{2} \geq 0.1\right)$ was observed mainly in residues in loop L5 (D192 and V198). Interestingly, this region is located in the vicinity of the phosphate group of 6PGA, although it does not directly interact with it. Note that loop L5 exhibits limited structural fluctuations on ligand binding. ${ }^{11}$

Residues R77 and R200, located near the active site, interact directly with the phosphate group of 6PGA. R77 shows an important decrease in rigidity $\Delta S^{2} \sim-0.19$, whereas for R200 no statistically significant MF parameters could be extracted from the data. For residue H165, which is actively involved in catalysis, the values of the order parameters in both forms of the protein were not significantly different and $\Delta S^{2} \sim 0$.

Exchange contributions $R_{\mathrm{ex}}$ to transverse relaxation were extracted for a substantial number of residues in both apo- and holo-Tb6PGL ( 24\% and $\sim 21 \%)$. Large exchange rates $R_{\mathrm{ex}}>10 \mathrm{~s}^{-1}$ [Figs. 2(c) and 3(b)] were observed in holo-Tb6PGL (I28, A41, T51, R77, V155, L158, and D133), and, to a lesser extent, in apo-Tb6PGL (I24, N87, and Q210).

In the holo-form, exchange rates $R_{\mathrm{ex}}$ holo may arise either from chemical shift perturbations caused by displacements of the ligand (which may be slipping in and out of the active site, leading to a bona fide case of chemical exchange) or from conformational exchange induced by the ligand. The occurrence of exchange on ligand binding for residues located in regions that are remote from the active site and do not interact with 6PGA indicate alterations of conformational exchange. This is the case for residues I28, A41, T51, V155, and L158, for which no $R_{\mathrm{ex}}$ was observed in the free protein. For residues A41, D154, V155, L158, R212, N213, V214, and N250, the rates $R_{\mathrm{ex}}$ holo are increased in the complex and seem to be clustered in an interfacial region of the $\beta$-sheet core [Fig. 3(b)]. Thus the presence of 6PGA seems to affect slow motions throughout the protein backbone.

In contrast, for residues located near the ligand, exchange contributions $R_{\mathrm{ex}}$ cannot always be unambigu- 

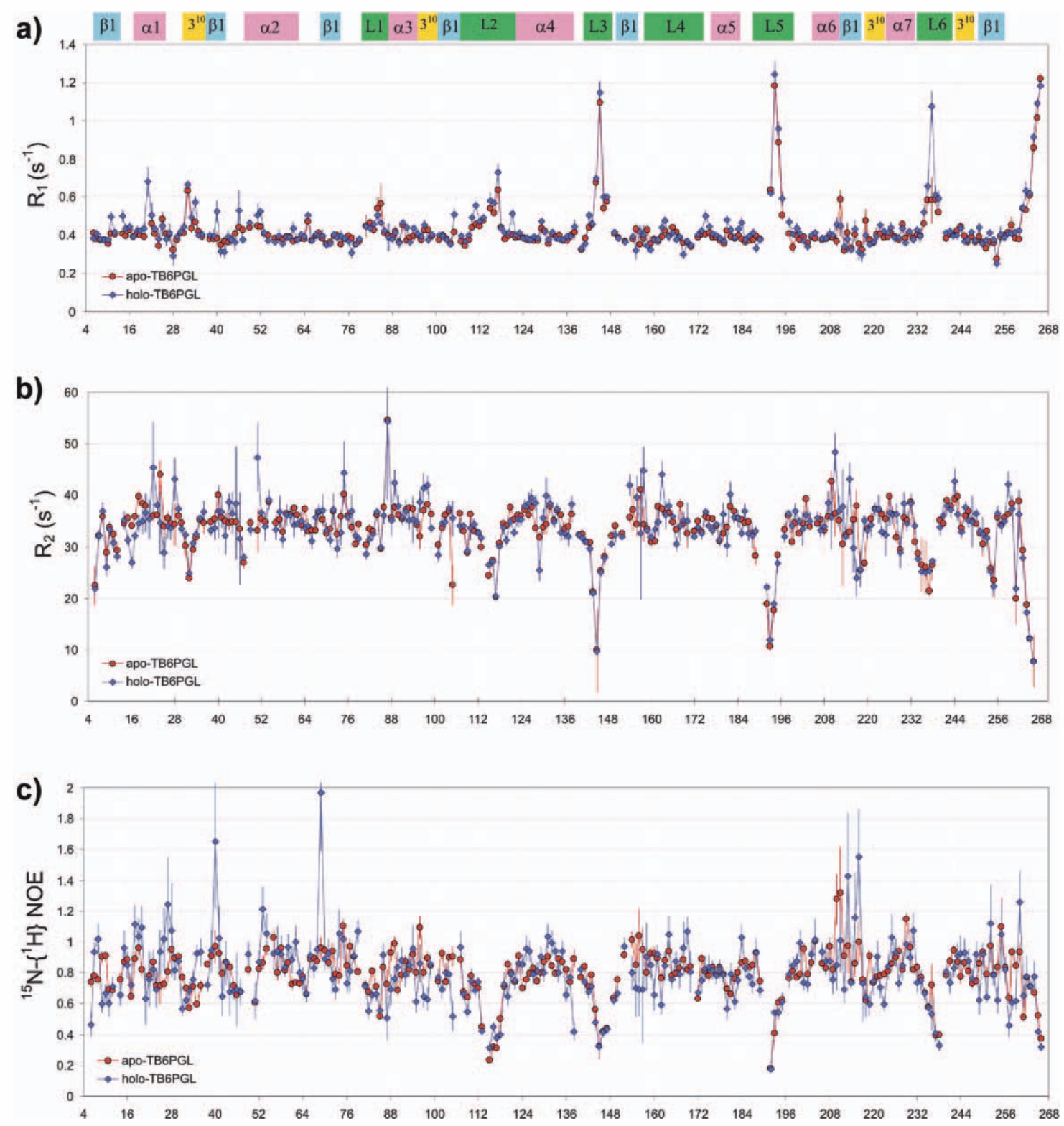

\section{Figure 1}

Longitudinal $\left(R_{1}\right)$ and transverse $\left(R_{2}\right)$ relaxation rates and ${ }^{15} \mathrm{~N}-\left\{{ }^{1} \mathrm{H}\right\} \mathrm{NOE}$ factors displayed in panels $(\mathbf{a})-(\mathbf{c})$. Secondary structure elements are indicated as colored boxes above the graphics. The $\alpha$ helices are found in the fragments $\alpha 1$ (13-31); $\alpha 2$ (46-61); $\alpha 3$ (87-93); $\alpha 4$ (120-137); $\alpha 5(174-177) ; \alpha 6$ (205-210); $\alpha 7$ (224-231); 310 helices (33-35; 96-98; 101-103; 221-223; 240-246), one parallel $\beta$ sheet $\beta 1$ (6-10, 38-42, 69-73, $104-105,155-158,213-218,250-256)$; two antiparallel $\beta$-sheets $\beta 2(140-142,149-151)$ and $\beta 3(75-77,185-188,200-203)$. The loops are L1 (7886), L2 (106-119), L3 (143-148), L4 (159-173), L5 (190-199), and L6 (233-238). The $R_{2}$ rates lie in the intervals $7.8 \pm 0.31<R_{2}{ }^{\text {apo }}<54.5 \pm$ $13.4 \mathrm{~s}^{-1}$ and $7.8 \pm 0.06<R_{2}^{\text {holo }}<54.5 \pm 15.3 \mathrm{~s}^{-1}$. The $R_{1}$ rates are distributed within the ranges $0.275 \pm 0.017<R_{1}$ apo $<1.22 \pm 0.026 \mathrm{~s}^{-1}$ and $0.25 \pm 0.026<R_{1}{ }^{\text {holo }}<1.25 \pm 0.067 \mathrm{~s}^{-1}$. The average ${ }^{15} \mathrm{~N}-\left\{{ }^{1} \mathrm{H}\right\}$ NOE factors are $\eta^{\text {apo }}=0.79 \pm 0.14$ and $\eta^{\text {holo }}=0.79 \pm 0.13$. [Color figure can be viewed in the online issue, which is available at wileyonlinelibrary.com.]

ously ascribed to chemical or conformation exchange. The exchange rate may be due, at least in part, to the off-rate of 6PGA. Indeed, assuming a dissociation constant $K_{\mathrm{d}}$ on the order of $10^{-4}-10^{-5} \mathrm{M}$ (V. Stoven and E. Miclet, unpublished results), and a diffusion-controlled on-rate $\left(10^{8} \mathrm{Ms}^{-1}\right)$, one expects an off-rate on the order of $10^{3}-10^{4} \mathrm{~s}^{-1}$, which is compatible with the time scale of the dynamical processes observed here.
Although it may be difficult to attribute the large $R_{\mathrm{ex}}^{\text {holo }}$ of S168 to conformational exchange in the holoform (the distance between its amide $\mathrm{N}$ atom and the phosphate group of 6PGA is only $6.77 \AA$ ), the exchange rate $R_{\mathrm{ex}}^{\text {holo }}=6.5 \pm 1.6 \mathrm{~s}^{-1}$ of S168 in apo-Tb6PGL is consistent with observations that slow motions may be an intrinsic property of proteins involved in ligand binding and enzymatic activity. ${ }^{4,43}$ Residue D163, which acts 

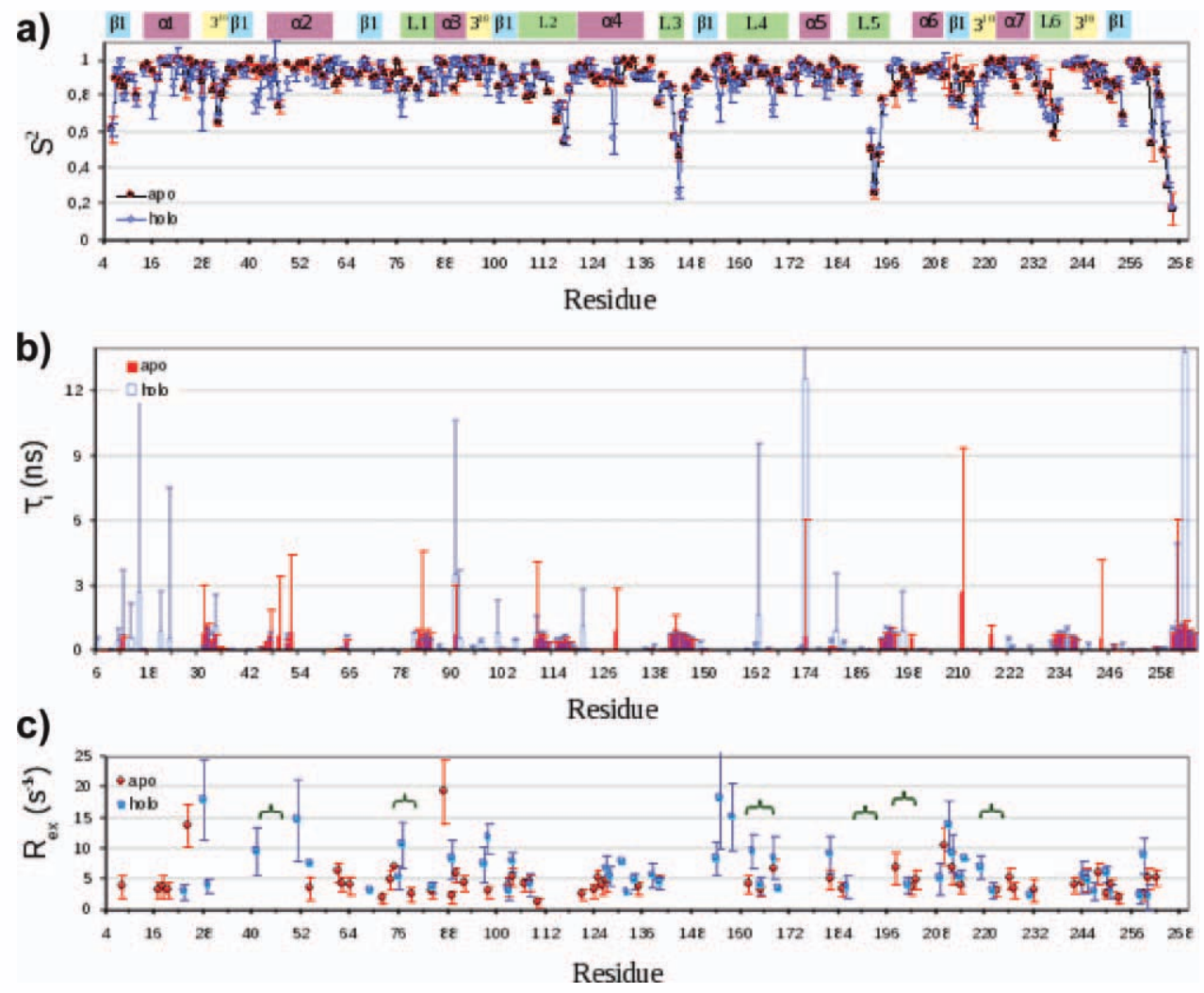

\section{Figure 2}

Model-free parameters in apo- and holo-Tb6PGL. The overall correlation time was $\tau_{c}=19.8 \pm 0.3 \mathrm{~ns}$. (a) Order parameters $S^{2}$, (b) internal correlation times $\tau_{\mathrm{i}}$, and (c) exchange contributions $R_{\text {ex }}$. Green brackets indicate the catalytic residue (H165) and residues R77 and R200 that bind directly to 6PGA The remaining marked regions contain residues G45, S46, T47, F170, M194, and K195 that exhibit direct contacts or hydrogen bonds with the ligand. [Color figure can be viewed in the online issue, which is available at wileyonlinelibrary.com.]

as a proton donor during catalysis, has an exchange rate $R_{\mathrm{ex}}{ }^{\text {holo }}=9.5 \pm 2.7 \mathrm{~s}^{-1}$. Although it belongs to the active site, it is rather distant from the ligand (its amide $\mathrm{N}$ and the phosphate group of 6PGA are $8.7 \AA$ apart), so that the $R_{\text {ex }}^{\text {holo }}$ can still be plausibly interpreted in terms of conformational exchange.

\section{Reduced SDM}

The dynamic analysis was complemented with the use of the reduced SDM approach $3,30,44$ (Fig. 4). Large values of $J_{\text {red }}(0)$ are usually associated with motions on a slow $\mu$ s-ms time scale, that is, chemical or conformational exchange, whereas fast ps-ns dynamics often lead to an increase of $J_{\text {red }}\left(\omega_{\mathrm{h}}\right)$ and a concomitant reduction of $J_{\text {red }}(0)$ and $J_{\text {red }}\left(\omega_{\mathrm{N}}\right) .44$ In both apo- and holo-proteins, we observed significantly higher values of $J_{\text {red }}\left(\omega_{h}\right)$ in loops L2, L3, L5, and L6, generally associated with values of $J_{\text {red }}(0)$ below the average. Changes of the reduced spectral density function $\Delta J_{\text {red }}(\omega)=J_{\text {red }}^{\text {holo }}(\omega)-$ $J_{\text {red }}{ }^{\text {apo }}(\omega)$ were observed on ligand binding. A significant increase of $J_{\text {red }}\left(\omega_{h}\right)$ was observed for about $20 \%$ of the residues, dispersed over the backbone, again suggesting a rather diffuse effect of 6PGA binding on protein dynamics. Most of these residues belong to $\beta$-sheet structures $(\beta 1, \beta 2$, and $\beta 3)$, loops and regions loosely structured at the junction between secondary structure elements, in the C-terminal region of the protein or in the short $3_{10}$ helices. This is summarized in Table I. Alternatively, analysis of $J_{\text {red }}\left(\omega_{h}\right)$ revealed that a few residues in holoTb6PGL exhibit a decreased mobility at high frequencies. Interestingly, this takes place in regions that are scattered across the protein backbone (I7, S8, E26, Q35, W36, L62, V79, D85, M90, F107, A116, T166, T193, W199, R200, A221, E248, and E261), and are by no means localized in the vicinity of the active site. Similar features have been observed for conformational exchange. 14

In addition, some variations in mobility seemed to occur in certain secondary structure elements. Thus, an increase of $J_{\text {red }}\left(\omega_{\mathrm{h}}\right)$ was noted for several residues in helix $\alpha 3$ (Y88, N89, and R92), whereas a significant number of residues with decreased $J_{\text {red }}\left(\omega_{\mathrm{h}}\right)$ are located in helix $\alpha 4$ (M124, R125, V126, A129, K132, and Q133), suggesting that these helices undergo a global change in fast dynam- 


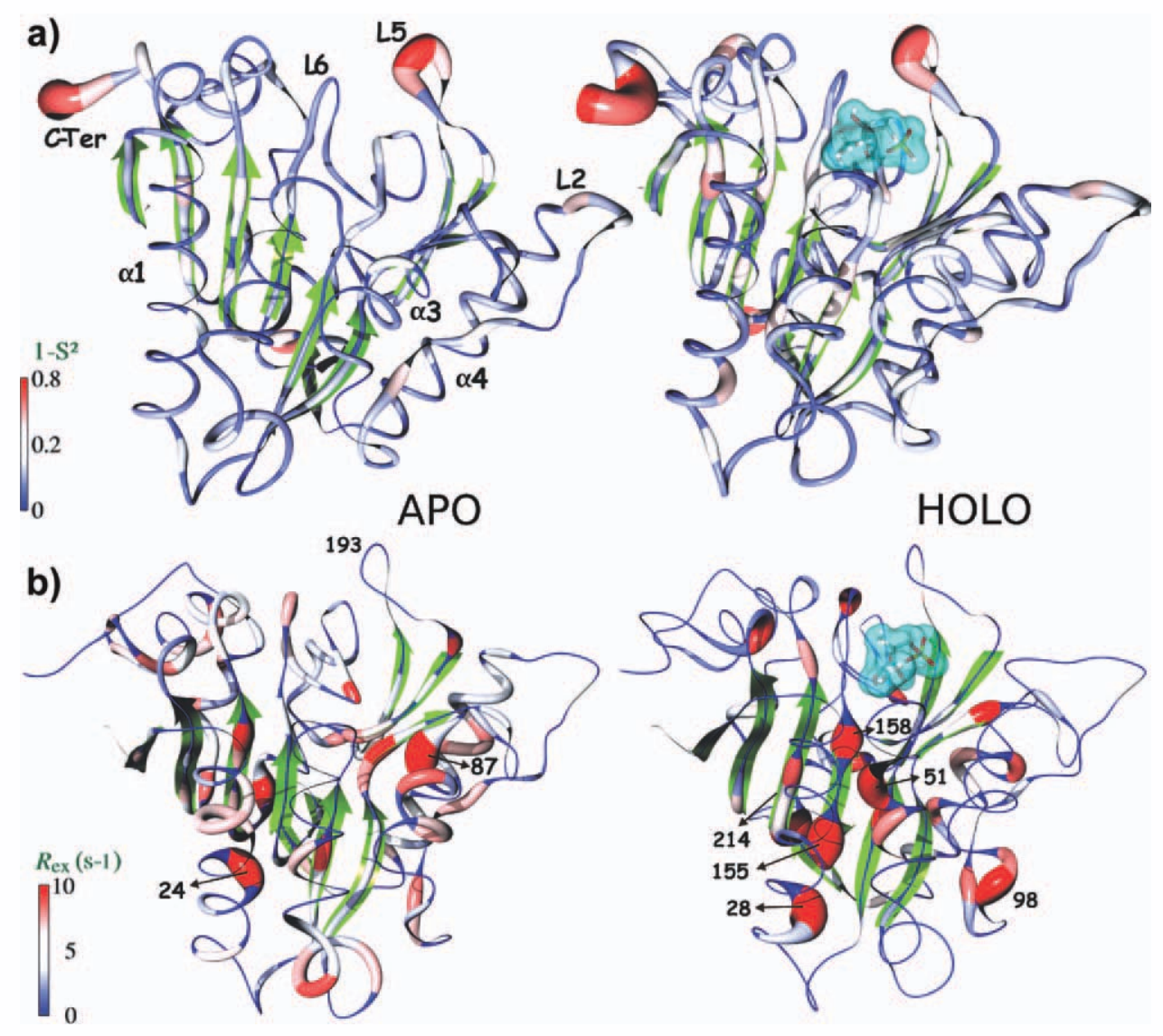

Figure 3

Results from the model-free analysis are mapped onto the 3D structure of 6PGL ( $\alpha$-helices and $\beta$-sheets colored in green and yellow). Apo- and holo-Tb6PGL are, respectively, depicted on the left and right sides of the figure. The ribbon thickness is proportional to $\left(1-S^{2}\right)$ in (a) and to $R_{\mathrm{ex}}$ in (b). Prolines and other unobservable residues are depicted in black. [Color figure can be viewed in the online issue, which is available at wileyonlinelibrary.com.]

ics on ligand binding. Remarkably, the relaxation rates of the latter residues could not be satisfactorily fitted by a MF approach, probably because the spectral density functions are too complex. Nevertheless, this analysis seems to indicate an increase of $J_{\text {red }}(\omega)$ values at frequencies likely lower than $\omega_{\mathrm{H}}$, yet detected by relaxation, therefore in the fast time scale. Interestingly, the side chains of the residues in helix $\alpha 4$ that are affected by ligand binding point toward the solvent. The dynamics of the other residues in this helix are not altered. This may be interpreted as evidence of less restricted dynamics in the protein-solvent interface that becomes allowed on ligand binding.

\section{Comparison with MD simulations}

A combination of NMR relaxation measurements and MD simulations helps to clarify global and local dynamic changes in 6PGL on ligand binding. Relaxation rates computed from our $77 \mathrm{~ns}$ MD simulations were found to be comparable to those obtained by NMR for most residues in both apo- and holo-forms when Eq. (8) was ful- filled (Fig. 5). In addition, order parameters were extracted from the trajectories and their variations between apoand holo-proteins examined. The presence of the ligand seems to have a significant effect on certain residues, mainly those located in loops. Thus, for residues A30 (in the transition region between helix $\alpha 1$ and the first $3^{10}$ helix) and A111-T113 (loop L2) one observes $\Delta S^{2} \leq-0.1$, whereas residues G144 and E145 (loop L3), and K195 (loop L5) experience increased rigidity $\left(\Delta S^{2} \geq 0.1\right)$. Discrepancies with NMR may be due to rare dynamical events that affect the averaging process. ${ }^{45}$ In addition, the root-mean-square-fluctuations (RMSFs) in the active site of apo- and holo-Tb6PGL are below $0.1 \AA$, suggesting both an overall rigidity of this region of the protein and a very limited effect of ligand binding.

The interatomic distances between the ligand and residues $\{\mathrm{G} 44, \mathrm{G} 45, \mathrm{~K} 223\}$ and $\{\mathrm{R} 77, \mathrm{R} 200\}$ in the active site reflect interactions with the carboxylate and phosphate groups of the ligand, respectively. In the former set, the carboxylate carbon of 6PGA is closer to the $\mathrm{N}^{\delta}$ atom of the K223 side chain than to the $\mathrm{C}^{\alpha}$ atoms of either G44 

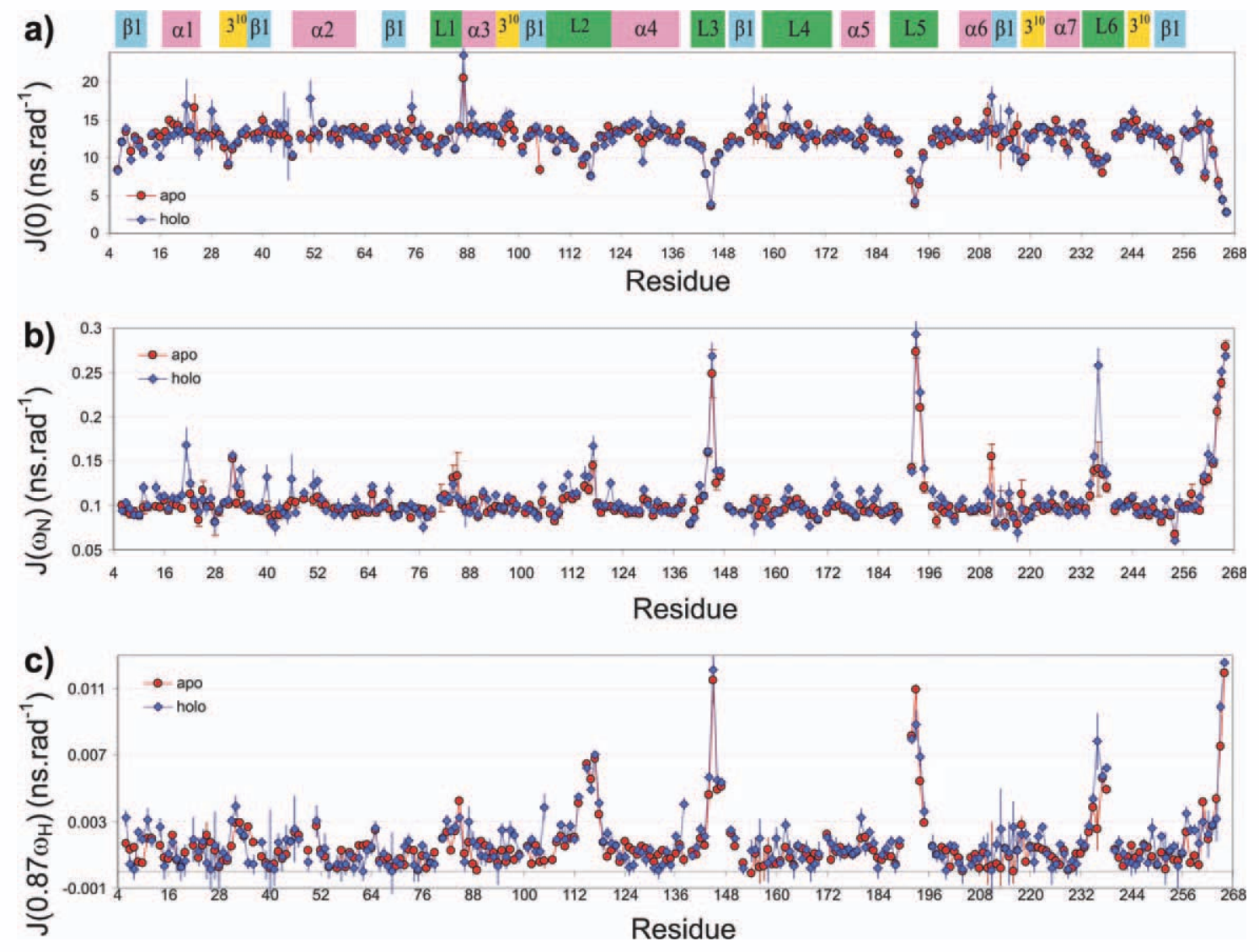

\section{Figure 4}

Reduced spectral density functions for apo- (red) and holo-Tb6PGL (blue), calculated from the relaxation rates shown in Figure 1. The spectral density function was sampled at $\omega=0, \omega_{\mathrm{N}}=91.14$, and $\omega_{\mathrm{h}}=0.87 \omega_{\mathrm{H}}=782.52 \mathrm{MHz}$ (for a proton resonance frequency 899.45 MHz. [Color figure can be viewed in the online issue, which is available at wileyonlinelibrary.com.]

or G45 during $80 \%$ of the MD trajectory, with average distances of about $5 \AA$ and $9 \AA$, respectively. These large distances make the presence of hydrogen bonds between the ligand and these residues very unlikely. Our results suggest that the carboxylate group of the ligand remains close to $\mathrm{K} 223$, as initially proposed by Delarue et al., 5 but in contrast with more recent work by the same authors, ${ }^{11}$ who concluded from the study of the X-ray structure of the 6PGA-Tb6PGL complex that the carboxylate is more likely to interact with the backbone atoms of G44 and G45 than with the side chain of K223. However, because our MD simulations started from the X-ray structure, where the carboxylate group of 6PGA is closer to residues G44 and G45, both conformations remain plausible and the ligand may adopt either of two positions alternatively.

As for dynamics, no significant changes in mobility could be deduced from NMR experiments for G44, G45, and K223 in the holo-protein, due to large errors in the MF parameters. However, MD simulations showed an increase of rigidity $\left(\Delta S^{2}\right.$ ca. 0.1$)$. Moreover, in some cases, angular autocorrelation functions of $\mathrm{NH}$ vectors
Table I

Residues Featuring Significative Changes of Dynamic Parameters in Going From apo- to holo-Tb6PGL Obtained From NMR Relaxation Experiments

\begin{tabular}{|c|c|c|c|}
\hline & $\Delta J\left(0.87 \omega_{H}\right)>0$ & $\Delta S^{2}>0$ & $\Delta S^{2}<0$ \\
\hline$\beta 1$ & $\begin{array}{l}\text { T6, V9, H10, A11, L38, } \\
\text { V104, F105, G154, R212, } \\
\text { V216, T219, T251, L254 }\end{array}$ & S8 & A41, V155, L217 \\
\hline$\beta 2$ & V150 & & \\
\hline$\beta 3$ & V187, V201 & & \\
\hline L1 & E76, D82, S83 & & E76, R77 \\
\hline L2 & T109, A111, T113, A118 & & \\
\hline L3 & L138, K141-G144, A146 & & E145 \\
\hline L4 & L160, S162, S173 & & S168 \\
\hline L5 & M194 & D192, V198 & \\
\hline L6 & A235, H236, A238 & & A235 \\
\hline$\alpha 1$ & 014, S31, G32 & & L16 \\
\hline$\alpha 3$ & & M90 & \\
\hline C-terminus & G249, K257-I259, K265, F266 & & \\
\hline $3^{10}$ helices & H97, D98, K223, A241, R242 & & \\
\hline Other & F72, L95, D102, D180, L182 & & \\
\hline
\end{tabular}

computed from MD simulations clearly exhibited different behavior in apo- and holo-6PGL. For instance, the 

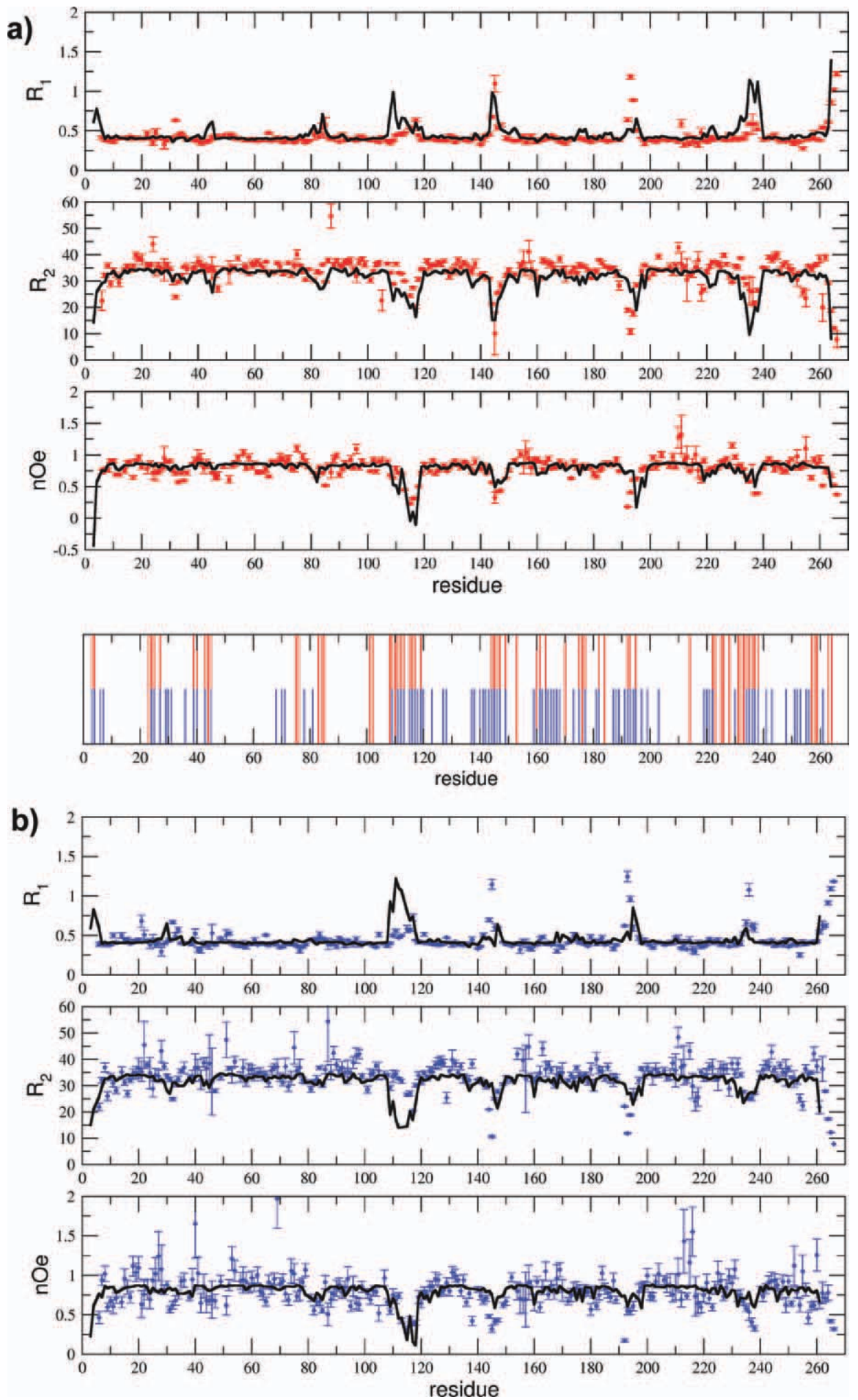

Figure 5

Experimental (points with error bars) and predicted (heavy line) NMR relaxation rates for (a) apo- and (b) holo-Tb6PGL. Residues for which the internal correlation functions did not reach a plateau after MD simulations running up to $77 \mathrm{~ns}$ are indicated in the central graph (red for apoand blue for holo-Tb6PGL). Most of the discrepancies between experimental and molecular dynamics NMR relaxation rates correspond to these residues. [Color figure can be viewed in the online issue, which is available at wileyonlinelibrary.com.] 


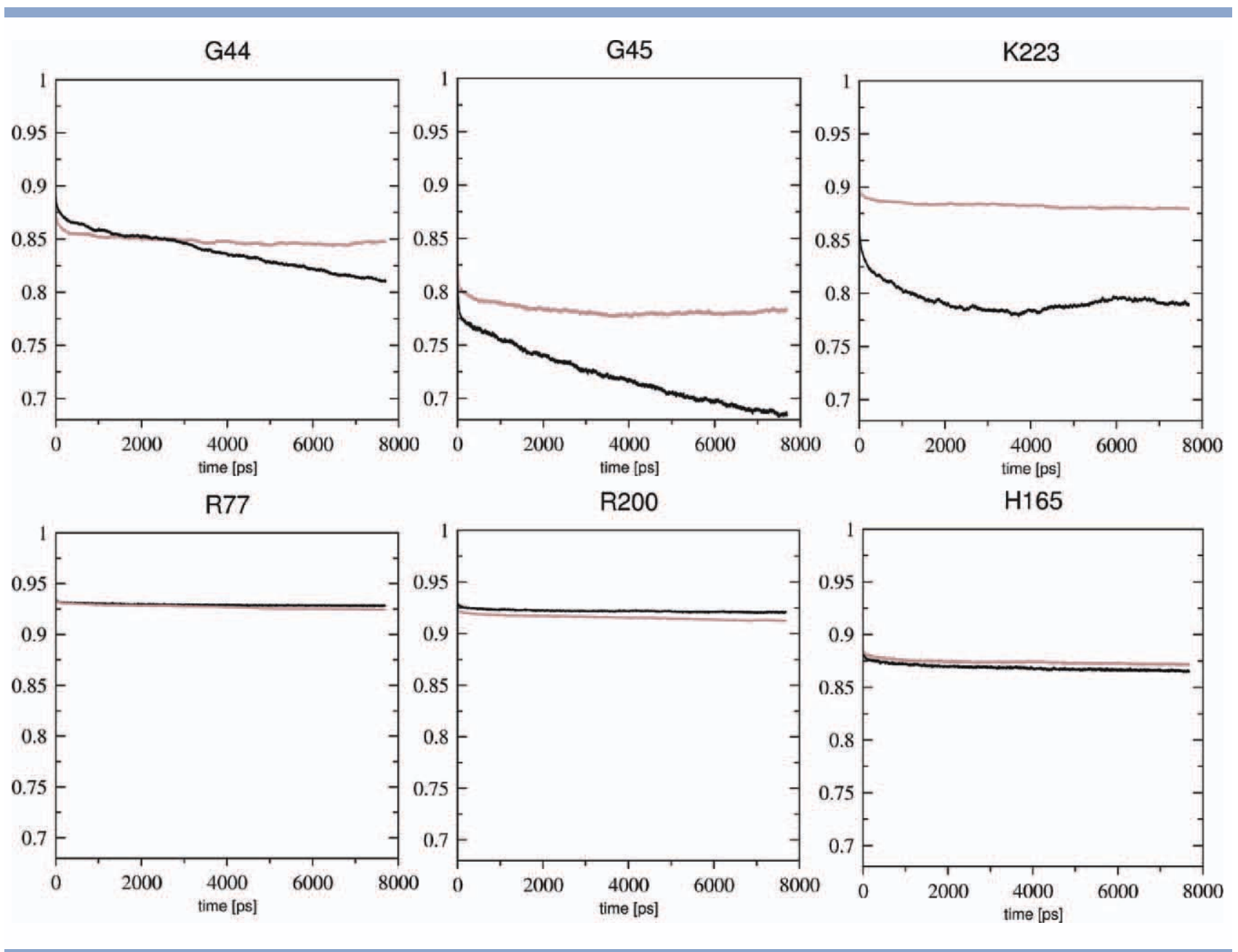

Figure 6

Internal correlation functions computed from MD simulations running up to $77 \mathrm{~ns}$ for selected residues of the active site in (black) apo- and (maroon) holo-Tb6PGL (see text for details). [Color figure can be viewed in the online issue, which is available at wileyonlinelibrary.com.]

correlation functions of residues G44, G45, and K223 did not reach a plateau during the MD runs for the apo-protein, whereas they clearly approached their asymptotic $S^{2}$ value for the holo-protein (see Fig. 6). This indicates a change toward spatially more restricted and faster motions induced by ligand binding. Similar results for K223 were also confirmed by SDM, where the absence of any variation of $J_{\text {red }}(0)$, associated with $\Delta J_{\text {red }}\left(\omega_{\mathrm{h}}\right)>0$ and $\Delta J_{\text {red }}\left(\omega_{\mathrm{N}}\right)>0$, must be ascribed to a significant exchange rate $R_{\mathrm{ex}}$ (confirmed by MF) that leads to an artificial overestimation of $J_{\text {red }}(0)$ in the apo-protein.

For the second group of residues $\{$ R77, R200\}, distances between hydrogen atoms of the guanidinium groups and the closest oxygen atom in the phosphate group of 6PGA remain in the range of hydrogen bond lengths (shorter that $3.0 \AA$ over nearly $90 \%$ of the MD trajectory). This can be related to previous studies ${ }^{11}$ suggesting that the phosphate group of the natural substrate $\delta$ 6-phosphogluconolactone is stabilized by R200 and R77.
Note that the distances between R77 or R200 on the one hand, and the phosphate group of 6PGA on the other hand, are shorter than those between the same residues and the phosphate group of $\delta$-6-phosphogluconolactone, which are larger than $3.0 \AA$ on average. ${ }^{11}$ This observation may indicate the presence of rearrangements of the reaction product in the catalytic site.

NMR data show that residue R77 exhibits an increased mobility on both fast and slow time scales $\left(\Delta S^{2}<0\right.$ and $\Delta R_{\text {ex }}>0$, without any significant variation of $J(\omega)$. The apparent discrepancy with MD, where only a slight decrease of mobility could be reproduced, as seen through $\Delta S^{2}$ and $J(\omega)$ (Fig. 6), is likely to be ascribed to internal motions on multiple time scales. A detailed investigation of these effects would require longer MD simulations. The MF parameters could not be obtained for R200 but the decrease of the high-frequency component $J_{\text {red }}\left(\omega_{\mathrm{h}}\right)$ in the presence of 6PGA indicates a redistribution of the motional energy toward lower frequen- 
cies in the complex without, however, any significant change of motion restriction $\left(\Delta S^{2}=0.01\right.$ in MD). This is interesting, as R200 is located near the N-terminal end of helix $\alpha 4$ and both seem to share these dynamical features (see above).

In this context, it should be noted that the phosphate ions of the buffer could compete with the phosphate group of 6PGA. However, the X-ray structures were obtained in the presence of $0.1 \mathrm{M}$ ammonium phosphate, and no phosphate was found to bind any residue in the binding pocket. Although a competition between buffer phosphate ions and the phosphate group of 6PGA cannot be ruled out, this should not alter our qualitative conclusions, which are essentially based on a comparison between apo- and holo-forms of the protein.

Neither NMR nor MD of the catalytic residue H165 and residue D163, which is believed to activate the former, ${ }^{5,11}$ (Fig. 6) revealed any significant alterations of the backbone amide group dynamics between the apoand holo-forms, whereas both MF and SDM indicate a significant increase of slow motions of D163 in holoTb6PGL (vide supra). However, H165 side-chain motions can be monitored by comparing the scalar product of the vector $\mathbf{r}(t)$ parallel to the $\mathrm{C} \beta-\mathrm{C} \gamma$ bond with its initial value $\mathbf{r}(t=0)$ (Fig. S3 in Supporting Information). Clearly, ligand binding significantly reduces the extent of H165 side-chain motions without affecting the backbone.

Residue S168, which is conserved in all proteins of the 6PGL family, ${ }^{11}$ points toward the phosphate group of the ligand and is thought to contribute to its stabilization. ${ }^{7,11}$ A moderate $S^{2}$ decrease in MD simulations $\left(\Delta S^{2}=-0.1\right)$ and MF analysis $\left(\Delta S^{2}=-0.16\right)$ show that mobility increases on binding to 6PGA. No significant change of $J_{\text {red }}(0)$ was observed, although $J_{\text {red }}{ }^{\text {apo }}(0)$ and $J_{\text {red }}$ holo $(0)$ were well above the average, in agreement with the $R_{e x}$ contributions in apo- and holo-proteins.

Besides residues in the binding site, dynamical changes on ligand binding occur across the protein backbone, as was already noticed from relaxation measurements. In this regard, significant effects appear to involve $\alpha 4$ and $\alpha 3$ helices. Moreover, the rigidity of residues M78-D85 in loop L1 that flank the active site is increased, as attested by MD order parameters and RMSF, as well as by experimental order parameters $\left(\Delta S^{2}=0.08\right.$ for A81, $\Delta S^{2}=0.06$ for D85). These effects are negligible for most other residues in L1. Such observations may be related to the presence of hydrogen bonds between R77 and N87. 5

In summary, the combination of several complementary methods for analyzing NMR relaxation data turned out to be beneficial. SDM could provide useful information when a MF analysis was not conclusive, and apparent contradictions could be explained. These results were supported by MD simulations (in particular for residues H165, D163, S168 in the active site, and others in helix $\alpha 4$ and loop L5). When no definite conclusions could be drawn from NMR relaxation, MD could provide indications of dynamical changes (R200). However, several discrepancies persisted between experiments and simulations. Nevertheless, even using rather conservative criteria for the significance of differences between holoand apo-forms of the protein, some of the discrepancies could be explained (G44, G45, and K223).

Additional information regarding the effect of ligand binding on the overall dynamic properties of the protein was obtained by an Essential Dynamics (ED) analysis of the MD trajectories of both proteins. ${ }^{46-50}$ This approach allows one to investigate the presence of collective atomic fluctuations in the protein, through diagonalization of the covariance matrix (of $\mathrm{C}_{\alpha}$ atoms in our case) and the determination of the eigen-directions of collective motions.

The distribution of eigenvalues are rather similar in apo- and holo-Tb6PGL, therefore indicating similar overall flexibilities in both proteins [see Fig. 7(a)]. This is confirmed by very similar Lindemann's flexibility indices $(\sim 0.19) .{ }^{51}$ Moreover, $90 \%$ of the total position fluctuations are contained in the first 72 modes in apo- and 80 modes in Tb6PGL suggesting the presence of limited but significant motional collectivity.

The first eigenvalue from holo-Tb6PGL was found to be significantly larger than the others in holo-Tb6PGL and also larger than its counterpart in the apo-form. ED eigenvalues represent atomic mean-square displacements. They indicate that motions involving the first eigenvector largely dominate over those encompassing the others in holo-Tb6PGL. They also suggest that these motions have larger amplitudes in holo-Tb6PGL than in the apo form.

Besides, the projections of the coordinate vectors corresponding to each snapshot of the MD trajectories onto the first two eigenvectors of apo-Tb6PGL show differences between atomic fluctuations occurring in both proteins. Thus, the rather limited overlap of these projections, shown in Figure 7(b), indicates slight but significant differences in fluctuations between the proteins.

Figure $7(\mathrm{c})$ shows a pictorial representation of the projections of the first motional modes onto the structures of apo- and holo-Tb6PGL. Interestingly, in holo-Tb6PGL, the largest eigenvalue involves an overall motion of the $\alpha 4$ helix and of loop L5, which is of larger amplitude than in the apo-protein. Moreover, the projection on the first eigenvector of the holo-Tb6PGL coordinate vector has significantly larger atomic root-mean-square-deviations in $\alpha 4$ and L5 regions $(\sim 2.75 \AA)$ than in the entire structure $(\sim 1.62 \AA)$. This is clearly in agreement with the conclusions drawn from the experimental NMR relaxation measurements performed in this study.

Finally, a comparison of RMSFs of the first two modes on $\mathrm{C} \alpha$ atoms [Fig. 7(d)] shows that differences are mostly located in the regions S110-L134 and V184V198, which, respectively, comprise residues of helix $\alpha 4$ and loop L5. The same observable suggests lower flexibility of loop L1 in holo-Tb6PGL. These findings again con- 
a)

b)
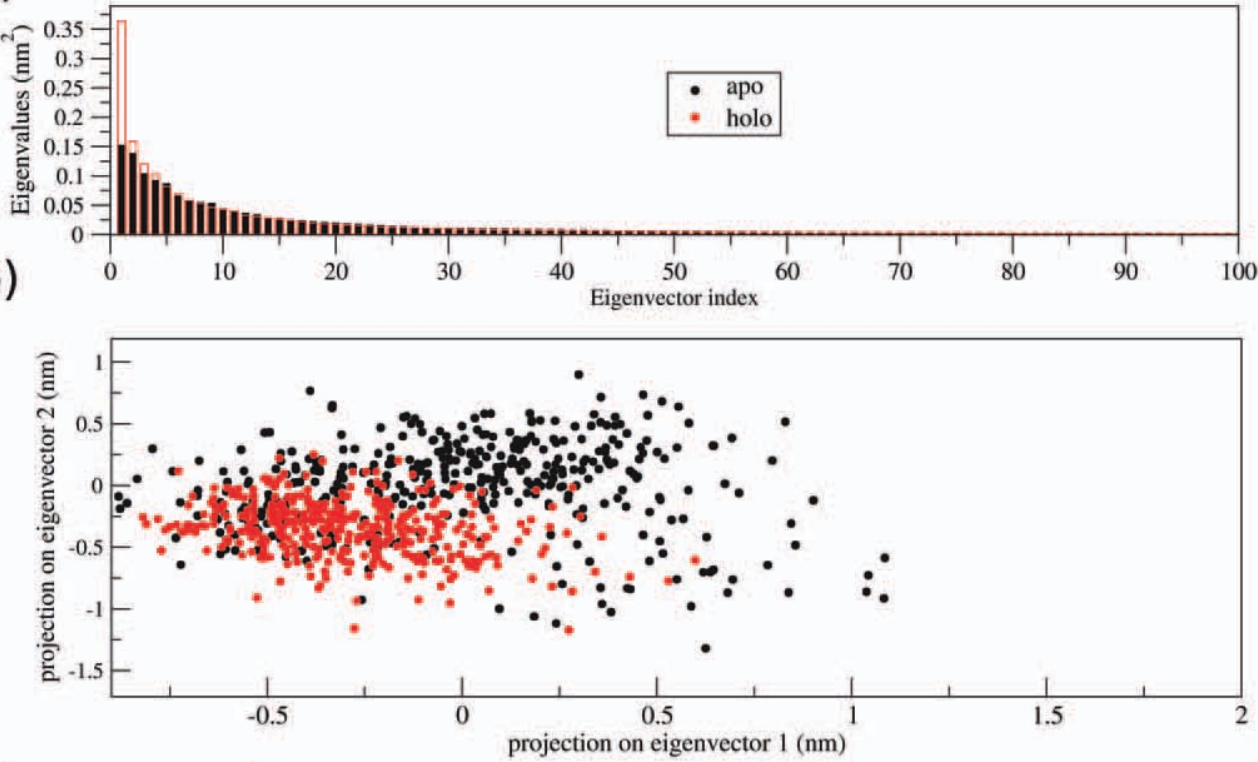

c)

d)
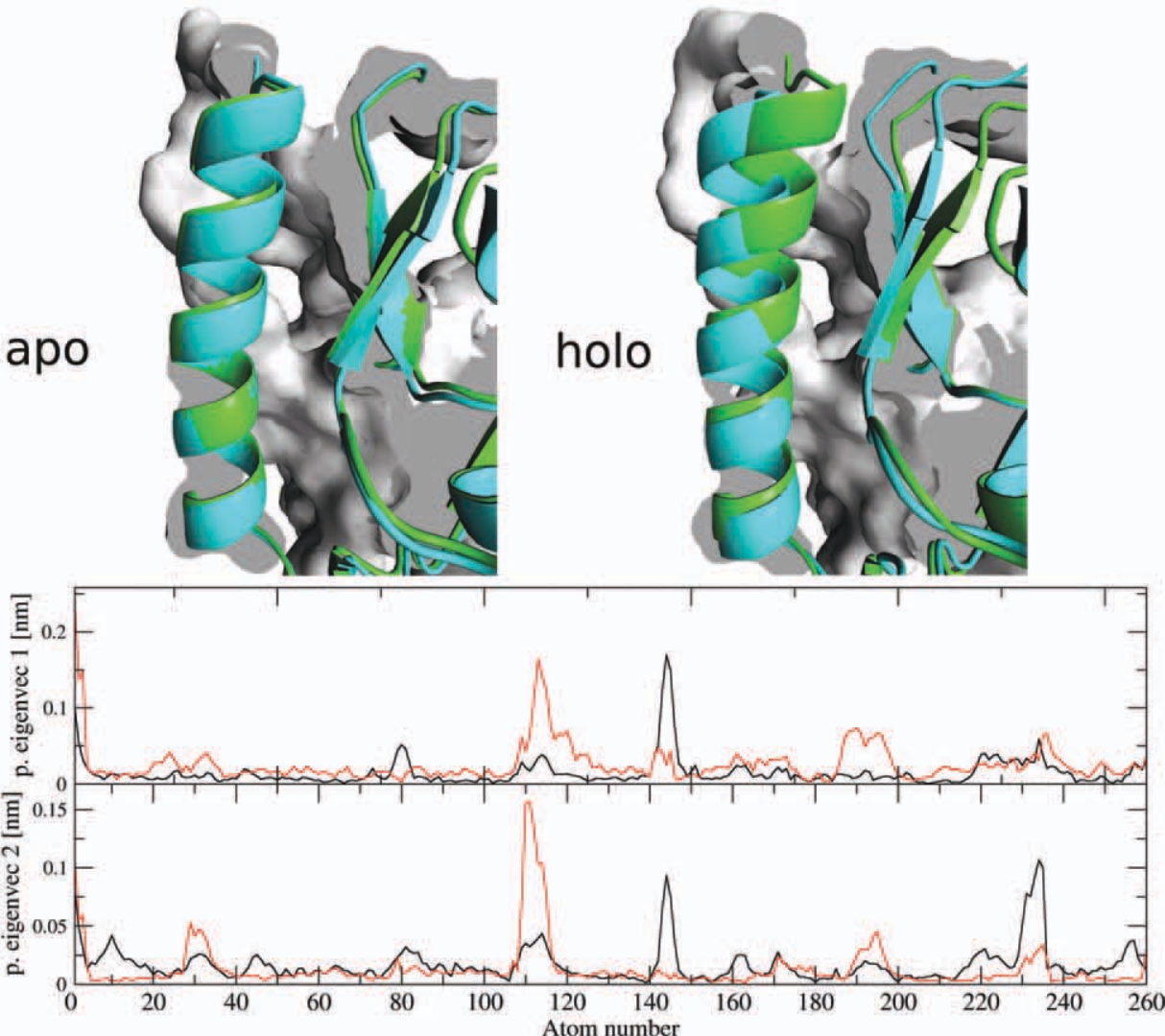

Figure 7

PCA analysis of apo- and holo-Tb6PGL trajectories. (a) PCA eigenvalues of apo- (black) and holo- (red) Tb6PGL; (b) projections of the coordinate vector of apo- (black) and holo- (red) onto the first two PC eigenmodes; (c) extreme deviations of the helix $\alpha 4$ and loop L5 along the first eigenmode for apo- (left) and holo- (right) Tb6PGL; (d) RMSF of the first two modes calculated for $\mathrm{C}_{\alpha}$ atoms in apo- (black) and holo- (red) proteins.

firm MD and NMR order parameter analyses presented above and strengthen the suggested pattern of dynamical changes dispersed over regions flanking the active site as well as remote ones. Although apparently significant contributions of the first two eigenmodes of apo-Tb6PGL for some residues in loops L3 and L6 in Figure 7(d) suggest 
the presence of higher flexibility, simulation data were not used in this case. Indeed, incomplete convergence of MD simulations of holo-Tb6PGL for these residues, as mentioned above, precludes further analysis and, a fortiori, a comparison with apo-Tb6PGL. These limitations are due to insufficient sampling of dynamical processes requiring simulation times longer than our 77 ns-long simulation. This leads to ill-averaged correlation functions, as illustrated above for a selection of other residues and also gives rise to large fluctuations of the estimates of the order parameters computed over several fractions (on the order of $10 \mathrm{~ns}$ ) of the total MD simulation (not shown). Not surprisingly, the ED analysis of fragments of the trajectory also exhibited large variances of the contributions of the first eigenmodes in the L3 and L6 loop regions. However, these were associated with much smaller fluctuations of the first eigenmode in $\alpha 4$ and L5. It was therefore concluded that poor MD convergence in the former region did not significantly affect the contributions of the first ED eigenmode in the latter, hence did not affect the foregoing analysis.

The repartitioning of internal mobility on ligand binding is a remarkable aspect of 6PGL dynamics. That the alteration of internal motions may affect residues that are located either close to the binding site or remote from it has only been described in a small number of NMR studies involving protein backbone $12,14,52$ or side-chain 53 relaxation experiments. On the other hand, a great deal of work has focused on the functional role of dynamics in enzyme-ligand interactions, and its relationship to structure and structural changes (e.g., allostery). ${ }^{54}$ Structure-dynamics relationships investigated through simple network models suggest that the dynamics is to a large extent encoded in the geometry of the protein. ${ }^{55-58}$ In addition, intrinsic dynamics in the apo state of an enzyme can determine structural changes occurring on ligand binding. ${ }^{59,60}$ In close connection, it has also been argued that motions that occur during catalysis may already be present in the free form and therefore represent an intrinsic functional property of the enzyme. ${ }^{61}$

The present comparative study also sheds light on a somewhat different but related aspect of dynamics. Indeed, our experiments reveal small local variations of relaxation parameters between the apo- and holo-forms of the protein. But analysis of MD simulations [see Fig. 7(a)] indicates that, in the holo-form in particular, most of these motions are contained in a single (first) mode. Therefore, it seems that it is in the holo-form that collective motions are more clearly present, which could be related to interactions with the ligand. Dynamic changes in Tb6PGL include a concerted increase in mobility in the $\alpha 4$ and L5 regions on binding of the reaction product. Together with an increased rigidity in loop L1 in the holo-form, which is likely related to the presence of hydrogen bonds, it seems that these changes might attest for both the release and binding of the reaction product.

\section{CONCLUSIONS}

We used NMR relaxation and MD simulations to study the alteration of internal dynamics of the protein upon binding to the reaction product. This provided information on the way internal molecular motions are redistributed when the protein accommodates the reaction product, that is, after the catalytic reaction has taken place (insight into the catalytic mechanism itself would require the use of a nonhydrolysable analogue of the substrate). Our observations indicate that the alterations of dynamics are not localized near the active site, but propagate to remote regions of the protein. The experimental facts gathered in this study, and their analysis, represent further compelling arguments in favor of a redistribution of internal protein mobility upon binding. Probing side-chain dynamics in Tb6PGL through ${ }^{15} \mathrm{~N}$ and ${ }^{13} \mathrm{C}$ NMR spectroscopy, ${ }^{62}$ as reported for other protein-ligand binding interactions, 63,64 would help to complement the picture.

\section{ACKNOWLEDGMENTS}

The authors thank Dr. Fabio Calogiuri and Dr. Isabella Felli for assistance at CERM (University of Florence), and to Dr. Fabien Ferrage for his critical comments. NMR measurements acquired at CERM in Florence were made possible through the EUNMR Contract \# RII3-026145. This work was granted access to the HPC resources of [CCRT/CINES/IDRIS] under grant 2010-x2010076423 awarded by GENCI (Grand Equipement National de Calcul Intensif).

\section{REFERENCES}

1. Daniel RM, Dunn RV, Finney JL, Smith JC. The role of dynamics in enzyme activity. Annu Rev Biophys Biomol Struct 2003;32:6992.

2. Hammes GG. How do enzymes really work? J Biol Chem 2008;283:22337-22346.

3. Jarymowycz VA, Stone MJ. Fast time-scale dynamics of protein backbones: NMR relaxation methods, applications, and functional consequences. Chem Rev 2006;106:1624-1671.

4. Boehr DD, Dyson HJ, Wright PE. An NMR perspective on enzyme dynamics. Chem Rev 2006;106:3055-3079.

5. Delarue M, Duclert-Savatier N, Miclet E, Haouz A, Giganti D, Ouazzani J, Lopez P, Nilges M, Stoven V. Three dimensional structure and implications for the catalytic mechanism of 6-phosphogluconolactonase from Trypanosoma brucei. J Mol Biol 2007;366:868881.

6. Miclet E, Stoven V, Michels PAM, Opperdoes FR, Lallemand JY, Duffieux F. NMR spectroscopic analysis of the first two steps of the pentose-phosphate pathway elucidates the role of 6-phosphogluconolactonase. J Biol Chem 2001;276:34840-34846.

7. Nowicki MW, Tulloch LB, Worralll L, McNae IW, Hannaert V, Michels PAM, Fothergill-Gilmore LA, Walkinshaw MD, Turner NJ. Design, synthesis and trypanocidal activity of lead compounds based on inhibitors of parasite glycolysis. Bioorg Med Chem 2008;16:5050-5061.

8. Duschak VG, Couto AS. An insight on targets and patented drugs for chemotherapy of Chagas disease. Recent Pat Antiinfect Drug Discov 2007;2:19-51. 
9. Kita K, Shiomi K, Omura S. Advances in drug discovery and biochemical studies. TRENDS Parasitol 2007;23:223-229.

10. Matsumoto J, Sakamoto K, Shinjyo N, Kido Y, Yamamoto N, Yagi K, Miyoshi H, Nonaka N, Katakura K, Kita K, Oku Y. Anaerobic $\mathrm{NADH}$-fumarate reductase system is predominant in the respiratory chain of Echinococcus multilocularis, providing a novel target for the chemotherapy of alveolar echinococcosis. Antimicrob Agents Chemother 2008:164-170.

11. Duclert-Savatier N, Poggi L, Miclet E, Lopes P, Ouazzani J, Chevalier N, Nilges M, Delarue M, Stoven V. Insights into the enzymatic mechanism of 6-phosphogluconolactonase from Trypanosoma brucei using structural data and molecular dynamics simulation. J Mol Biol 2009;388:1009-1021.

12. MacRaild CA, Daranas AH, Bronowska A, Homans SW. Global changes in local protein dynamics reduce the entropic cost of carbohydrate binding in the arabinose-binding protein. J Mol Biol 2007;368:822-832.

13. Zídek L, Novotny MV, Stone MJ. Increased protein backbone conformational entropy upon hydrophobic ligand binding. Nat Struct Mol Biol 1999;6:1118-1121.

14. Perazzolo C, Wist J, Loth K, Poggi L, Homans S, Bodenhausen G. Effects of protein-pheromone complexation on correlated chemical shift modulations. J Biomol NMR 2005;33:233-242.

15. Frederick KK, Marlow MS, Valentine KG, Wand AJ. Conformational entropy in molecular recognition by proteins. Nature 2007;448:325-329.

16. Kamerlin SC, Warshel A. At the dawn of the 21st century: is dynamics the missing link for understanding enzyme catalysis? Proteins 2010;78:1339-1375.

17. Pervushin K, Riek R, Wider G, Wuthrich K. Attenuated T2 relaxation by mutual cancellation of dipole-dipole coupling and chemical shift anisotropy indicates an avenue to NMR structures of very large biological macromolecules in solution. Proc Natl Acad Sci USA 1997;94:12366-12371.

18. Mandel AM, Akke M, Palmer AG. Backbone dynamics of Escherichia coli ribonuclease $\mathrm{HI}$ : Correlations with structure and function in an active enzyme. J Mol Biol 1995;246:144-163.

19. Kay L, Nicholson LK, Delagio F, Bax A, Torchia DA. Pulse sequences for removal of the effects of cross correlation between dipolar and chemical shift anisotropy relaxation mechanisms on the measurement of heteronuclear $\mathrm{T}_{1}$ and $\mathrm{T}_{2}$ values in proteins. J Magn Reson 1992;97:359-375.

20. Ferrage F, Piserchio A, Cowburn D, Ghose R. On the measurement of ${ }^{15} \mathrm{~N}-\left\{{ }^{1} \mathrm{H}\right\}$ nuclear Overhauser effects. J Magn Reson 2008;192:302-313.

21. Loria JP, Rance M, Palmer AG. Transverse-relaxation-optimized (TROSY) gradient-enhanced triple-resonance NMR spectroscopy, J Magn Reson 1999;141:180-184.

22. Weisemann R, Rueterjans H, Bermel HW. 3D triple-resonance NMR techniques for the sequential assignment of $\mathrm{NH}$ and ${ }^{15} \mathrm{~N}$ resonances in ${ }^{15} \mathrm{~N}$ - and ${ }^{13} \mathrm{C}$-labelled proteins. J Biomol NMR 1993;3:113-120.

23. Ikegami T, Sato S, Waelchli M, Kyogoku Y, Shirakawa M. An efficient $\mathrm{HN}(\mathrm{CA}) \mathrm{NH}$ pluse scheme for triple-resonance $4 \mathrm{D}$ correlation of sequential amide protons and nitrogens-15 in deuterated proteins. J Magn Reson 1997;124:214-217.

24. Delaglio F, Grzesiek S, Vuister GW, Zhu G, Pfeifer J, Bax A. NMRPipe: a multidimensional spectral processing system based on UNIX pipes. J Biomol NMR 1995;6:277-293.

25. Goddard TD, Kneller DG. SPARKY 3. University of California, San Francisco.

26. de la Torre JG, Huertas ML, Carrasco B. HYDRONMR: prediction of NMR relaxation of globular proteins from atomic-level structures and hydrodynamic calculations. J Magn Reson 2000;147:138-146.

27. Dosset P, Hus J-C, Blackledge M, Marion D. Efficient analysis of macromolecular rotational diffusion from heteronuclear relaxation data. J Biomol NMR 2000;16:23-28.

28. Clore GM, Szabo A, Bax A, Kay LE, Driscoll P, Gronenborn AM. J Am Chem Soc 1990;112:4989-4936.
29. Abragam A. The principles of nuclear magnetism. Oxford, UK: Clarendon Press; 1961.

30. Lefevre JF, Dayie KT, Peng JW, Wagner G. Internal mobility in the partially folded DNA binding and dimerization domains of GAL4: $\mathrm{NMR}$ analysis of the $\mathrm{N}-\mathrm{H}$ spectral density functions. Biochemistry 1996;35:2674-2686.

31. Phillips JC, Braun R, Wang W, Gumbart J, Tajkhorshid E, Villa E, Chipot C, Skeel RD, Kale L, Schulten K. Scalable molecular dynamics with NAMD. J Comput Chem 2005;26:1781-1802.

32. Hornak V, Abel R, Okur A, Strockbine B, Roitberg A, Simmerling C. Comparison of multiple Amber force fields and development of improved protein backbone parameters. Proteins 2006;65: $712-725$.

33. Wang J, Wolf RM, Caldwell JW, Kollman PA, Case DA. Development and testing of a general amber force field. J Comput Chem 2004;25:1157-1174.

34. Darden T, York D, Pedersen L. Particle mesh Ewald—an $\operatorname{Mlog}(N)$ method for Ewald sums in large systems. J Chem Phys 1993;98:10089-10092.

35. Ryckaert J, Ciccotti G, Berendsen H. Numerical integration of the Cartesian equations of motion of a system with constraints: molecular dynamics of n-alkanes. J Comput Phys 1977;23:327-341.

36. Izaguirre JA, Catarello D., Wozniak JM, Skeel RD. Langevin stabilization of molecular dynamics. J Chem Phys 2001;114:2090-2098.

37. Nosé S. Constant temperature molecular-dynamics methods. Prog Theor Phys Supp 1991;103:1-46.

38. Chen J, Brooks CL, III, Wright PE. Model-free analysis of protein dynamics: assessment of accuracy and model selection protocols based on molecular dynamics simulation. J Biomol NMR 2004;29:243-257.

39. Zwanzig R, Ailawadi NK. Statistical error due to finite time averaging in computer experiments. Phys Rev 1969;182:280.

40. Miclet E, Duffieux F, Lallemand J-Y, Stoven V. Backbone HN, N, $\mathrm{C} \alpha, \mathrm{C}^{\prime}$, and $\mathrm{C} \beta$ assignment of the 6-phosphogluconolactonase, a 266-residue enzyme of the pentose-phosphate pathway from human parasite Trypanosoma brucei. J Biomol NMR 2003;25:249-250.

41. Kay LE, Torchia DA, Bax A. Backbone dynamics of proteins as studied by nitrogen-15 inverse detected heteronuclear NMR spectroscopy: application to staphylococcal nuclease. Biochemistry 1989;28:8972-8979.

42. Lipari G, Szabo A. Model-free approach to the interpretation of nuclear magnetic resonance relaxation in macromolecules. 1. Theory and range of validity. J Am Chem Soc 1982;104:45464559.

43. Henzler-Wildman KA, Lei M, Thai V, Kerns SJ, Karplus M, Kern D. A hierarchy of time-scales in protein dynamics is linked to enzyme catalysis. Nature 2007;450:913-916.

44. Farrow NA, Zhang O, Szabo A, Torchia DA, Kay LE. Spectral density function mapping using $15 \mathrm{~N}$ relaxation data exclusively. J Biomol NMR 1995;6:153-162.

45. Philippopoulos M, Mandel AM, Palmer III AG, Lim C. Accuracy and precision of NMR relaxation experiments and MD simulations for characterizing protein dynamics. Proteins 1997;28: 481-493.

46. Amadei A, Linssen A, Berendsen H. Essential dynamics of proteins. Proteins 1993;17:412-425.

47. Laine E, Yoneda JD, Blondel A, Malliavin T. The conformational plasticity of calmodulin upon calcium complexation gives a model of its interaction with the oedema factor of Bacillus anthracis. Proteins 2008; 71:1813-1829.

48. Kubitzki MB, de Groot BL. Molecular dynamics simulations using temperature-enhanced essential dynamics replica exchange. Biophys J 2007;92:4262-4670.

49. Hayward S, de Groot BL. Normal modes and essential dynamics. Methods Mol Biol 2008;443:89-106.

50. de Groot BL, Daura X, Mark AE, Grubmüller H. Essential dynamics of reversible peptide folding: memory-free conformational 
dynamics governed by internal hydrogen bonds. J Mol Biol 2001; 309:299-313.

51. Zhou Y, Vitkup D, Karplus M. Native proteins are surface-molten solids: application of the Lindemann criterion for the solid versus liquid state. J Mol Biol 1999;285:1371-1375.

52. Zidek L, Novotny MV, Stone MJ. Increased protein backbone conformational entropy upon hydrophobic ligand binding. Nat Struct Biol 1999;6:1118-1121.

53. Lee AL, Kinnear SA, Wand AJ. Redistribution and loss of side chain entropy upon formation of a calmodulin-peptide complex. Nat Struct Biol 2000;7:72-77.

54. Bahar I, Chennubhotla C, Tobi D. Intrinsic dynamics of enzymes in the unbound state and relation to allosteric regulation. Curr Opin Struct Biol 2007;17:633-640.

55. Bahar I, Atilgan AR, Erman B. Direct evaluation of thermal fluctuations in proteins using a single parameter harmonic potential. Fold Des 1997;2:173-81.

56. Eyal E, Yang LW, Bahar I. Anisotropic network model: systematic evaluation and a new web interface. Bioinformatics 2006;22:26192627.

57. Dhulesia A, Abergel D, Bodenhausen G. Networks of coupled rotators: relationship between structures and internal dynamics in metal-binding proteins. Applications to apo- and holo-Calbindin, J Am Chem Soc 2007;129:4998.
58. Dhulesia A, Bodenhausen G, Abergel D. Predicting conformational entropy of bond vectors in proteins by networks of coupled rotators. J Chem Phys 2008;129:095107.

59. Bakan A, Bahar I. The intrinsic dynamics of enzymes plays a dominant role in determining the structural changes induced upon inhibitor binding. Proc Natl Acad Sci 2009;106:14349-14354.

60. Gunasekaran K, Ma B, Nussinov R. Is allostery an intrinsic property of all dynamic proteins? Proteins 2004;57:433-443.

61. Eisenmesser EZ, Millet O, Labeikovsky W, Korzhnev DM, WolfWatz M, Bosco DA, Skalicky JJ, Kay LE, Kern D. Intrinsic dynamics of an enzyme underlies catalysis. Nature 2005;438:117-121.

62. Mulder FA, Skrynnikov NR, Hon B, Dahlquist FW, Kay LE. Measurement of slow $(\mu \mathrm{s}-\mathrm{ms})$ time-scale dynamics in protein side chains by ${ }^{15} \mathrm{~N}$ relaxation dispersion $\mathrm{nmr}$ spectroscopy: application to Asn and Gln residues in a cavity mutant of T4 lysozyme. J Am Chem Soc 2001;123:967-975.

63. Paquin R, Ferrage F, Mulder FA, Akke M., Bodenhausen G. Multiple-time-scale dynamics of side-chain carboxyl and carbonyl groups in proteins by ${ }^{13} \mathrm{C}$ nuclear spin relaxation. J Am Chem Soc 2008;130:15805-15807.

64. Hass MA, Yilmaz A, Christensen HE, Led JJ. Histidine side-chain dynamics and protonation monitored by 13C CPMG NMR relaxation dispersion. J Biomol NMR 2009;44:225-233. 